ÉTUDES

IIIII 



\title{
Paris et ses banlieues dans les romans de Faïza Guène et Rachid Djaïdani
}

\author{
Paris and its Suburbs (banlieues) \\ in Faïza Guène and Rachid Djaïdani’s Novels
}

\author{
Mirna Sindičıć SABlJo [msindici@unizd.hr] \\ Sveučilište u Zadru, République de Croatie
}

\section{RESUMÉ}

Dans son ouvrage La capitale des signes. Paris et son discours (2002), Karlheinz Stierle souligne que Paris est la première grande ville moderne à avoir engendré un grand mythe urbain cohérent, dynamique et riche en perspectives. Crystel Pinçonnat et Chantal Liaroutzos dans Paris, cartographies littéraires (2007) montrent le caractère profondément hétérogène de la capitale et de son imaginaire. Renvoyant à la " géocritique » (Westphal 2000 ; 2007) et à la métaphore spatiale de la « cartographie littéraire » (Pinçonnat - Liaroutzos 2007), en guise de méthode qui convient à l'étude d'un espace urbain hétérogène sur lequel se pose une multiplicité de regards focalisateurs, nous proposons une étude d'identification des espaces de lisibilité de la ville dans quelques romans contemporains d'auteurs français issus de l'immigration : Faïza Guène (Kiffe kiffe demain, 2004 ; Du rêve pour les oufs, 2006 ; Les gens du Balto, 2008) et Rachid Djaïdani (Boumkoeur, 1999 ; Mon nerf, 2004 ; Viscéral, 2007). Ce travail vise à analyser des représentations littéraires que Guène et Djaïdani ont offertes de Paris et de sa banlieue dans leurs romans. Le rapport à l'espace urbain est une constante dans la vie des personnages de ces romans, souvent d'origine immigrée. Les images spécifiques de la capitale et de ses banlieues dans les romans de Guène et Djaïdani contribuent aux transformations de conscience de la ville et contestent l'image mythifiée de Paris.

\section{MOTS-CLÉS}

espace urbain ; centre / périphérie ; banlieue ; Paris ; immigration

\begin{abstract}
In his book La capitale des signes. Paris et son discours (2002) Karlheinz Stierle explains that Paris is the first big modern city that has generated a coherent urban myth. That myth is dynamic and abounds in different perspectives. Crystel Pinçonnat and Chantal Liaroutzos in their book Paris, cartographies littéraires (2007) point out to the profoundly heterogeneous character of the French capital and its imagery. Relying on the spatial metaphor of the "cartographie littéraire" (Pinçonnat - Liaroutzos 2007) and the geocriticism (Westphal 2000; 2007), this paper deals with the literary representation of Paris and its suburbs (banlieues) in the novels written by the two French writers of immigrant origin: Faïza Guène (Kiffe kiffe demain, 2004 ; Du rêve pour les oufs, 2006 ; Les gens du Balto, 2008) and Rachid Djaïdani (Boumkoeur, 1999; Mon nerf, 2004; Viscéral, 2007). The complex relationship with the urban space in which the action is taking place is
\end{abstract}


a constant in the life of the characters. The novels express the sense of the marginalization of the habitants of the banlieues within the French society. By presenting the communal spirit and affiliation, they refute the stereotypes of the banlieues as place of violence and crime. The images of Paris and its suburbs in the novels of Djaidani and Guène contribute to the transformation of the mythic image of Paris.

\section{KEYWORDS}

urban space; centre/periphery; suburbs; Paris; immigration

REÇU 2018-01-07 ; ACCEPTE 2018-02-25

\section{Introduction}

L'espace urbain de Paris ne cesse de susciter l'intérêt des écrivains depuis le Moyen Âge. Paris est la première grande ville moderne à avoir généré un mythe urbain cohérent, dynamique et riche en perspectives diverses, comme le signale Karlheinz Stierle dans son ouvrage La capitale des signes. Paris et son discours (1993, traduction française 2002). ${ }^{1}$ Stierle explique que la ville se reflète elle-même et accède à la conscience dans son mythe, ou dans son discours, comme une totalité de l'expérience (2002 : 561). Jusqu'à aujourd'hui, le mythe de Paris est resté le mythe urbain par excellence, même si, depuis, maintes grandes villes dans le monde ont développé leur propre mythe. À la création du mythe de la ville de Paris participent les essais éphémères et anonymes des dilettantes et des journalistes tout autant que les grandes œuvres dans lesquelles l'esprit de la ville parvient à sa plus profonde révélation. Tous ces textes de la ville font partie du grand palimpseste de la ville. L'histoire du discours de Paris est une histoire de la complexité croissante des formes de représentation se construisant les unes sur les autres, capables de pénétrer toujours plus profondément dans la complexité réelle de la ville et de sa conscience (Stierle $2002: 562$ ).

Paris, pensé comme un mythe, a tendance à être représenté comme un tout homogène. Cependant, Crystel Pinçonnat et Chantal Liaroutzos dans Paris, cartographies littéraires (2007) montrent le caractère profondément hétérogène de la capitale et de son imaginaire. Le livre qu'elles ont édité met en avant une pluralité des perspectives. Conséquemment, le mythe de Paris éclate pour faire place à une succession de regards. Les articles recueillis dans ce livre offrent un Paris pluriel et font percevoir l'hétérogénéité qu'exhibe la littérature.

En renvoyant à la métaphore spatiale de la cartographie littéraire (Pinçonnat-Liaroutzos 2007) et à la " géocritique » (Westphal 2000 ; 2007), comme méthodes d'approche qui conviennent à l'étude d'un espace urbain hétérogène, ce travail vise à identifier les espaces de lisibilité de

1 Roger Caillois était un des premiers à considérer que la littérature au cours du XIX ${ }^{\mathrm{e}}$ siècle a fabriqué un mythe de Paris. Ce mythe est sans cesse renouvelé et toujours changeant. Les auteurs qui contribuent au renforcement de Paris comme mythe littéraire sont, selon Caillois, Honoré de Balzac, Eugène Sue, Alexandre Dumas, Victor Hugo et Charles Baudelaire (Caillos 1938 : 150-171). Pierre Citron considère qu'un vaste mythe de Paris sélaborait à partir de 1830 ( $L a$ poésie de Paris dans la littérature française de Rousseau à Baudelaire, Paris, 1961). Selon Patrice Highonnet, les mythes ne sont pas l'évocation d'un passé perdu, mais la stylisation d'une réalité quotidiennement vécue. Ils ne sont pas uniformes dans le temps (1999: 11). 
la ville dans les romans de Faïza Guène (Kiffe kiffe demain, 2004 ; Du rêve pour les oufs, 2006 ; Les gens du Balto, 2008) et de Rachid Djaïdani (Boumkoeur, 1999 ; Mon nerf, 2004 ; Viscéral, 2007). L'étude sera centrée sur l'analyse de l'image de Paris et de ses banlieues ${ }^{2}$ mise en place par ces romanciers français issus de l'immigration. ${ }^{3}$ Nous proposerons d'abord une description de Paris et de ses banlieues dans les romans de Guène et Djaïdani. Enfin, nous mettrons ces images en perspective.

Les romans de Rachid Djaïdani et de Faïza Guène construisent une image spécifique de la capitale et de ses banlieues. Ce faisant, ils ajoutent de nouvelles perspectives au mythe littéraire de Paris. Ce travail vise à démontrer que les romans en question nous invitent à concevoir une nouvelle perception de l'espace en remettant en question les représentations externes des territoires des banlieues, souvent réduits à un type précis d'habitat urbain peuplé en majorité d'étrangers. ${ }^{4}$ Les romans démontrent l'hétérogénéité des banlieues ainsi que le rapport dynamique et dialectique qu'entretient le centre (Paris) avec sa périphérie (les banlieues).

Les romans de Rachid Djaïdani et de Faïza Guène mettent en scène des espaces romanesques qui interagissent fortement avec le réel, tout en apportant une contribution à ce que la géocritique désignera par une "théorie des mondes ». Selon Bertrand Westphal, la théorie des mondes présente un intérêt évident dans le cadre d'une analyse des représentations littéraires de l'espace (Westphal 2007 : 162). Les propositions théoriques de la "géocritique» (Westphal 2000 ; 2007) nous permettront de concevoir l'étude de l'espace urbain comme « celui des interactions entre espaces humains et littérature ». Selon Westphal, l'objet de la géocritique n'est pas un examen des représentations de l'espace en littérature, mais plutôt celui des interactions entre espaces humains et littérature. Une nouvelle lecture orientera le lecteur vers une perception plurielle de l'espace, ou vers la perception des espaces pluriels. La géocritique correspondrait à une poétique de l'archipel, de l'espace dont la totalité est constituée par l'articulation raisonnée de tous les îlots qui le composent. La géocritique permettrait de percevoir en tout espace l'archipel qui le fonde. Elle se propose d'étudier non seulement une relation unilatérale (espace-littérature), mais aussi et de surcroît une véritable dialectique (espace-littérature-espace) qui implique le constat selon lequel l'espace se transforme à son tour en fonction du texte qui, antérieurement,

2 Étymologiquement, le terme «banlieue » a un sens juridique, puisque c'était l'espace à une lieue à la ronde autour des fortifications d'une ville, l'espace qui dépendait juridiquement de la ville avoisinante.

3 Faïza Guène est née en 1985 à Bobigny, en Seine-Saint-Denis, de parents algériens. Elle vit dans la cité des Courtillières, à Pantin (région parisienne) depuis l'âge de 8 ans. Son père est arrivé en France en 1952. Rachid Djaïdani est un comédien et écrivain français d’origine algéro-soudanaise, né dans la cité des Grésillons, à Carrières-sous-Poissy dans le département des Yvelines. Djaïdani et Guène sont considérés par les critiques littéraires comme faisant partie de 'la littérature de banlieue'. Cette notion, apparue dans les années 1990, unit des textes différents qui ont en commun la représentation de la vie quotidienne des jeunes dans les cités HLM. (voir Hargreaves 2014).

4 L'Observatoire national des zones urbaines sensibles, dans son rapport annuel de 2011, affirme que plus d'une personne sur deux $(52,6 \%)$ vivant dans les quartiers sensibles est issue de l'immigration. Le chiffre monte à 64\% dans les zones urbaines sensibles (ZUS) de la région parisienne. Les personnes issues de l'immigration sont plus souvent au chômage que le reste de la population. ZUS coïncide dans la majeure partie des cas avec ce que lon désigne communément par banlieues, cités, quartiers populaires (Frayer-Vincent 2011 : 11). Pourtant, il y a une confusion générale entre «banlieue ", terme général et « cité », terme spécifique. Ce dernier indique les quartiers des cités-ouvrières. Le glissement sémantique entre banlieue et cité est pourtant tellement courant que celles-là finissent par s'identifier à celles-ci. La banlieue n'est une entité ni géographique, ni urbanistique ni sociale homogène. Luc Bronner explique : « La banlieue n’existe pas. Ou, du moins, n'a pas de sens au singulier. Utilisons le pluriel : les banlieues, les riches, les moyennes, les pauvres, les proches, les lointaines, les bourgeoises, les pavillonnaires, les populaires, les chics, les "sensibles" ... (2010:32). 
l'avait assimilé. Les relations entre littérature et espaces humains ne sont donc pas figées, mais parfaitement dynamiques. L'espace transposé en littérature influe sur la représentation de l'espace dit réel (référentiel), sur cet espace-souche dont il activera certaines virtualités ignorées jusque-là, ou ré-orientera la lecture (Westphal 2000).

\section{Les banlieues dans les romans de Rachid Djaïdani et Faïza Guène}

Dans les romans beurs des années $1980^{5}$, les cités HLM (habitation à loyer modéré) de la banlieue parisienne sont le plus souvent décrites comme des endroits orduriers et froids dont les habitants sont les victimes de la pauvreté, du chômage, des échecs scolaires et de la violence. Les cités HLM dans les romans beurs se caractérisent d'abord par la monotonie des formes architecturales et la dégradation des immeubles. Les romans décrivent l'incommodité du cadre de vie dans des cités dépourvues de jardins, de centres d'éducation et de sport, de rues commerciales et de monuments. Ces romans témoignent aussi d'une séparation radicale entre centre (Paris) et sa périphérie (les banlieues). Le centre historique de Paris est à éviter pour les jeunes vivants dans des grands ensembles HLM de la banlieue qui n’ont pas les capacités nécessaires de participer aux activités commerciales, sociales, culturelles et politiques de la capitale. Paris est le lieu symbolique de leur exclusion de la société française dominante. La métaphore du milieu carcéral est une métaphore filée tout au long de ces romans. ${ }^{6}$ Il suffit de prendre comme exemple le roman Le thé au harem d'Archi Ahmed de Mehdi Charef (1983) afin de vérifier les affirmations précédentes. ${ }^{7}$

Les premiers romans de Rachid Djaïdani (Boumkoeur, 1999) et de Faïza Guène (Kiffe kiffe demain, 2004) continuent cette tradition, à un certain degré, et représentent les cités HLM de la banlieue, dans lesquelles se déroule l'action, comme des endroits sales et dévastés. Kiffe kiffe

5 Le terme « beur » est entré dans le langage courant de la population majoritaire à partir de 1983 et la Marche des beurs pour l'égalité et contre le racisme. Le terme a été créé en inversant l’ordre des syllabes du mot arabe. Les premières œuvres littéraires dues à cette nouvelle génération commencent à voir le jour pendant les années 1970. Létiquette « la littérature beur » inclut seulement les écrivains d’origine maghrébine, nés ou installés en France. Hargreaves explique que la littérature issue de l'immigration maghrébine est un corpus particulièrement difficile à designer et à classer. Il n’existe aucun consensus quant aux critères par lesquels le corpus se définirait, ni sur les appellations qu'il conviendrait de lui appliquer. L’année 1983 est aussi l'année de la publication du Thé au harem d’Archi Ahmed de Mehdi Charef, le plus connu parmi les romans beurs. Sur la littérature « beur », voir Hargreaves (1991).

6 Michel Laronde explique que l'architecture du modèle panoptique n'est jamais aussi évidente que dans la spatialité de la région parisienne. Autour d'un point central (Paris) s'est élargie une concentricité partout présente dans la terminologie territoriale : arrondissements, ceinture, périphérique, proche banlieue, grande banlieue, couronne. Paris et sa banlieue reproduisent géographiquement les unités du dispositif carcéral : grosso modo autour de Paris s'est développé un anneau qui héberge le plus grand nombre d'immigrés (1993: 96).

7 «La Cité des Fleurs, que ça s’appelle !!!

Du béton, des bagnoles en long, en large, en travers, de l'urine et des crottes de chiens. Des bâtiments hauts, longs, sans cœur ni âme. Sans joie ni rires, que des plaintes, que du malheur.

Une cité immense entre Colombes, Asnières, Gennevilliers, et l'autoroute de Pontoise et les usine set les flics. Le terrain de jeux, minuscule, ils l’ont grillagé !

Les fleurs! Les fleurs!

Et sur les murs de béton, des graffiti, des slogans, des appels de détresse, des S.O.S en forme de poing levé. » (Charef 1983 : 24-25) 
demain de Faïza Guène raconte l'histoire d'une banlieue au nord de Paris, Livry-Gargan, vue à travers les yeux de Doria, une fille de 15 ans qui y vit seule avec sa mère. Doria parle franchement et avec douceur du quotidien des gens qui l'entourent. Dans ce roman, l'environnement dans lequel se déroule l'action n'est pas plus agréable que dans les romans beurs :

Arrivés en bas de notre immeuble, Maman a remercié Youssef et puis il est reparti. Le gardien de nos immeubles, il s'en fout de l'état des tours on dirait. Heureusement que des fois Carla la femme de ménage portugaise nettoie un peu. Mais quand elle vient pas, ça reste bien dégueulasse pendant des semaines, comme là ces derniers temps. Dans l'ascenseur, y avait de la pisse et des mollards, ça sentait mauvais, mais on était quand même contentes que ça marche. Heureusement qu'on connaît l'emplacement des boutons par rapport aux étages, parce que la plaquette est grattée et ça a fondu. On a dû les bruler au briquet (Guène 2004 : 36-37).

Doria vit dans une tour HLM séparée d'une zone pavillonnaire par un mur qu’elle compare au mur de Berlin :

Il y a quand même une séparation bien marquée entre la cité du Paradis où j’habite et la zone pavillonnaire Rousseau. Des grillages immenses qui sentent la rouille tellement ils sont vieux et un mur de pierre tout le long. Pire que la ligne Maginot ou le mur de Berlin (Guène 2004 : 89-90).

Boumkoeur de Rachid Djaïdani prend les apparences d'une chronique de la vie d'une cité en banlieue. Le narrateur qui s'appelle Yaz a 21 ans et il vit avec sa famille dans l'une des tours de la cité. Lui aussi décrit sa cité comme un endroit sale :

Le porche est complétement abimé, abandonné. Les soins quotidiens du gardien ne se lustrent plus, celui-ci a démissionné. Les poubelles percées, la pisse et le sang se déchargent ici comme des champignons. Un porc ne pourrait vivre là sans avoir à craindre de se gober un mauvais microbe (Djaïdani 1999 : 20).

Yaz aussi se sent prisonnier de la cité qu’il trouve trop éloignée de la capitale :

Une galère de plus comme tant d'autres jours dans ce quartier où les tours sont tellement hautes que le ciel semble avoir disparu. Les arbres n'ont plus de feuilles, tout est gris autour de moi. Moi, c'est Yazad, mais dans le quartier on me surnomme Yaz. C'est mortel comme il caille, j'ai l'impression dêtre dans mon frigidaire. » (Djaïdani 1999 : 9)

Lies, protagoniste de Viscéral, troisième roman de Rachid Djaïdani (2007), évoque sa banlieue dans des termes similaires à ceux du narrateur dans Boumkoeur:

Les premiers rayons du soleil enflamment une cité immense, la plus cramée du territoire gaulois. Inaugurée il y a trente ans, elle n'a pas d'appellation contrôlée, pas de label, pas de millésime.

Ici les rats portent des combinaisons en Teflon. Les cafards font du smurf sur les os des mollards. Les pits sniffent des rails de coke avant de chiquer des têtards. Le béton a de l'herpès soigné au 
Kärcher, les barbelés le sida, et la Déclaration universelle des droits de l'homme est une blague qui circule sous le manteau. » (Djaïdani $2007: 7$ )

Ahlème, le protagoniste du roman $D u$ rêve pour les oufs qui habite dans une cité à Ivry avec son père et son frère décrit, d'ailleurs, sa banlieue de la même manière :

Je suis entourée par tous ces immeubles aux aspects loufoques qui renferment nos bruits et nos odeurs, notre vie d'ici. Je me tiens là, seule, au milieu de leur architecture excentrique, de leurs couleurs criardes, de leurs formes inconscientes qui ont si longtemps bercé nos illusions. Il est révolu le temps où l'eau courante et l'électricité suffisaient à camoufler les injustices, ils sont loin maintenant les bidonvilles. » (Guène 2006 : 29-30)

Pourtant, les romans de Guène et Djaïdani ne s'arrêtent pas à la description de l'isolement et de la dégradation architecturale des cités. Ils introduisent des représentations plus nuancées des cités HLM, ainsi que la représentation d'autres types des banlieues. ${ }^{8}$ Dans Mon nerf (2004) et Les Gens du Balto (2008) Djaïdani et Guène abandonnent les cités HLM comme lieux où se déroule l'action pour les zones pavillonnaires de la banlieue parisienne. Laction des Gens $d u$ Balto se déroule à Joigny-les-deux-Bouts, petite ville pavillonnaire en fin de la ligne RER. ${ }^{9}$ Mounir, protagoniste de Mon nerf, habite dans un pavillon à Bois-Fleury. Il n'a pas grandi dans une cité. ${ }^{10}$ Mounir évolue entre deux mondes, ne se sentant ni Parisien, ni faisant partie d'une communauté d'une cité HLM.

Dans leur description du quotidien, les narrateurs des romans de Djaïdani et Guène offrent une variété de portraits et de situations qui tranchent avec les stéréotypes sur la banlieue comme un lieu de violence, pauvreté et non-droit. Ces romans n'offrent pas seulement des visions négatives de l'endroit des cités HLM, ils évoquent aussi un attachement de la part des personnages pour leur cadre de vie. Ce que les romans en question ont en commun c'est de représenter les quartiers comme des communautés fraternelles. Les cités, soit HLM soit pavillonnaires, sont considérées de la part des personnages comme leur micro-patrie et elles sont représentées en tant que lieux d'une interaction sociale forte. Les quartiers sont des lieux de vie, de circulations et d'échanges entre les habitants. Les habitants des quartiers donnent vie à l'espace :

La cité est cartographiée comme un camp de bataille, les tours, les rues, les allées, les places ont été rebaptisées par les faits divers. La rue des Violettes est devenue la rue des Cinq-Vierges après

8 Christina Horvath souligne qu'il est intéressant de noter que dans les représentations littéraires, la banlieue se résume grosso modo aux cités. Dans son livre, elle affirme que les auteurs français contemporains trouvent apparemment peu d'intérêt pour les quartiers résidentiels occupés par la classe moyenne qui prévalent dans les littératures anglosaxonnes (2007: 46).

9 Joigny-les-deux-Bouts est « une commune de 4500 habitants à l'extrémité d'une ligne RER. Un endroit dans lequel vous ne foutrez sans doute jamais les pieds » (2008: 9). Taniel, un des personnages, dit à propos de Joigny : « Je sais même pas si on peut appeler ça une ville. Le marché sur la place, la mairie, La Poste, la pharmacie, la boulangerie, la gendarmerie, le Balto. C'est à peu près tout ce qu'il y a » (Guène 2008 : 14-15).

10 «La cité, malgré mon facies rocailleux, je ne l'ai jamais foulée du pied, à peine décortiquée du regard. Elle est située dans les hauteurs de la commune, non loin d'une bretelle d'autoroute et d'un lac pollué de poisson-chat. » (Djaïdani $2004: 25-26)$. 
la mort d'un groupe de jeunettes fauchées par un chauffard. La place du Rond-Point est transformée en place du Prématuré : une femme enceinte y a été poignardée par son mari et le bébé est venu au monde sur le trottoir. La tour des Castors s'est changée en tour Ben Omar, du nom d'un suicidé que la solitude et la détresse ont conduit à se jeter du haut de son bloc (Djaïdani $2007: 17)$.

Les quartiers des banlieues se découvrent de l'intérieur comme des quartiers vivants qui prennent forme à travers les personnages qui l'habitent. Les cités sont une microsociété où se développent des liens communautaires. Les habitants se connaissent et une solidarité s'installe entre eux. Par exemple, dans Kiffe kiffe demain, Rachida, comme tous les résidents du quartier, soutient matériellement Doria et sa mère, et l'épicier leur offre parfois des vivres. Nabil aide Doria avec ses devoirs tandis qu'elle garde la fille de Lila, une mère célibataire. Tante Zohra et une très bonne amie de la mère de Doria. Dans Viscéral, lorsqu'un proche meurt, tout le monde aide la famille. Mariatou remplace la mère absente d'Ahlème dans Du rêve pour les oufs et lui sert de soutien. Une solidarité très forte s'installe aussi entre les protagonistes de Viscéral dans leur tentative de créer une meilleure vie dans leur cité.

La cité, vue comme un village, est l'expression du sentiment de l'appartenance des habitants des grands ensembles à l'endroit dans lequel ils vivent. La cité est intimement appropriée et les personnages la réclament en tant qu'espace exclusivement privé. Les jeunes protagonistes des romans s'identifient à l'espace dans lequel ils vivent. À titre d'exemple, dans Viscéral, Teddy et Samir, les élèves de Lies, revendiquent avec fierté leur appartenance à la cité :

Teddy et Samir sont des graines qui ont poussé dans le huis clos du quartier. Frères de béton, ils savent ce qu'est l'injustice, le vise, et pratiquent l'art de la combine. Pour tout l'or du monde jamais ils n'abandonneraient leur cité téci tess, ils la revendiquent dans leur façon de respirer, de penser, de la raconter. La tess est la fondation de ce qu'ils sont, plutôt mourir que la quitter (Djaïdani $2007: 8$ ).

Les protagonistes des romans, tous issus de l'immigration, obtiennent une identité sociale en termes spatiaux. Ils se revendiquent jeunes de cités, en opposition aux jeunes de Paris et des quartiers aux pavillons. L'espace qu'ils hébergent détermine l'identité transnationale des protagonistes des romans. Par conséquent, les cités de la banlieue deviennent un laboratoire de nouvelles identités françaises hybrides et multiculturelles.

\section{Paris dans les romans de Rachid Djaïdani et Faïza Guène}

Paris est, physiquement, visuellement et symboliquement, séparé des départements de la petite couronne par les " portes » et par le boulevard "périphérique ». À la rupture géographique et territoriale marquée dans le paysage urbain s'ajoute une très forte rupture d'image et de puissance entre Paris, qui s'identifie au luxe, aux arts, à la culture et au raffinement, et ses voisins plus anonymes (Ronai $2004: 28$ ). 
Dans les romans beurs, Paris est une ville inaccessible, un lieu qui renforce le sentiment de non-appartenance des protagonistes résidants dans les cités HLM de la banlieue. ${ }^{11}$ Paris, bien que spatialement proche, s'est transformé en une ville lointaine pour ceux qui la regardent depuis les cités. Les protagonistes des romans beurs ne participent pas à la vie culturelle, économique, sociale ou politique du centre-ville. Prenons l'exemple du Thé au harem d'Archi Ahmed: Madjid et Pat ne vont à Paris que pour faire du pickpocket dans le métro ou pour fréquenter les prostituées à Strasbourg-Saint-Denis. Josette y cherche le travail sans succès et la sœur de Paty y va se prostituer. Selon Michel Laronde, les représentations du rapport entre Paris et ses banlieues dans les romans beurs reprennent la dichotomie centre-périphérie autrefois spécifique au rapport entre la Métropole et ses périphéries colonisées (1993 : 95-97).

Lopposition Paris / banlieue est omniprésente dans les romans de Rachid Djaïdani et Faïza Guène, mais la nature de leur rapport a changé des premiers aux derniers romans de ces écrivains. Paris n'existe pas dans Boumkoeur. Ce roman nie l'autorité de Paris puisque les personnages ne le mentionnent pas et n'y vont pas. Dans Boumkoeur, il n'existe donc ni comme un point de référence ni comme un point d'orientation.

Pour les personnages des Gens du Balto Paris est le lieu dans lequel ils veulent être. Nadia et Ali Chacal, les jumeaux marseillais récemment installés à Joigny-les-deux-Bouts, parlent de leur désillusion après le déménagement dans les environs de Paris :

Sincèrement, quand papa nous a dit qu’on montait à Paris, dans ma tête, j’ai vu la tour Eiffel, les boutiques, l'ambiance. J'ai pensé que ça allait être la fête et que la seule chose que j'allais regretter c'est la mer. En vrai, c'est pas Paris ici, c'est rien du tout. C'est la campagne, il faut une heure et demie de train pour toucher la capitale (p. 40).

Taniel, lui non plus, n'est pas content de la vie dans une banlieue pavillonnaire, qu'il considère d'ailleurs ennuyeuse par rapport à Paris :

Bref, j'avance. Si on est allés au Balto, c'est pas pour l'ambiance, c'est clair qu’on aurait préféré les Champs-Elysées (p. 68-69).

L'action des autres romans de Djaïdani et de Guène ne se déroule pas exclusivement entre les murs des cités ou des zones pavillonnaires. Les protagonistes se rendent plus fréquemment dans le centre de Paris, qui constitue l'une des échappatoires à la vie dans les grands ensembles HLM. Par exemple, Doria tente de s'extraire de son milieu grâce à des fugues vers Paris. Pour elle, Paris représente le lieu où il est possible de se balader librement. Lorsqu'elle s'ennuie, Doria parcourt la ligne 5 du métro d'un bout à l'autre. ${ }^{12}$ Elle conquit activement l'espace du centre de

11 Paris et sa banlieue sont en totale opposition dans le film La Haine, réalisé par Mathieu Kassovitz en 1995. Ce film est un des plus connus parmi les films faisant partie du cinéma de banlieue (voir Tarr 2005). Lorsque les trois personnages dans La Haine prennent le R.E.R. pour aller dans le centre de Paris, ils ressentent un sentiment d'exclusion totale, ce qui est perceptible surtout dans la scène dans laquelle ils pénètrent dans une gallérie d'art. Le déplacement à Paris dans La Haine est véritablement symptomatique de la distance qui peut exister entre le centre et la périphérie.

12 «Comme je m’ennuyais, j’ai décidé de prendre le métro. Je ne savais pas où aller mais je trouve ça distrayant le métro. On voit plein de têtes, c'est marrant. J'ai fait toute la ligne 5, terminus à terminus » (Guène $2004: 29$ ). 
Paris et c'est elle qui emmène sa mère, qui habite dans la banlieue parisienne depuis vingt ans, dans une promenade au centre historique de Paris :

Comme Mama est encore en vacances jusqu'à la semaine prochaine, on a décidé d'aller se balader toutes les deux dans Paris. La tour Eiffel, c'était la première fois quelle la voyait en vrai alors qu'elle habite à une demi-heure depuis vingt ans. Autrement, c'était à la télé, au JT de treize heures, le lendemain du jour de l'an quand elle est illuminée et qu'à ses pieds, des gens font la fête, dansent, s'embrassent et se bourrent la gueule. En tout cas, elle était vachement impressionnée.

—Ça doit faire peut-être deux ou trois fois notre bâtiment, non?

Je lui ai répondu que c'était surement ça. Sauf que notre immeuble et la cité en général, ils suscitent moins d'intérêt auprès des touristes. $\mathrm{Y}$ a pas des mafias de Japonais avec leur appareil photo au pied des tours du quartier. Les seuls qui s'y intéressent, c'est les journalistes mythos avec leurs reportages dégueulasses sur la violence en banlieue. (Guène 2004 : 125)

Mais, la tour Eiffel, symbole de Paris, accessible aux touristes venus du monde entier, demeure hors de portée de Doria et de sa mère qui n’habitent pourtant qu'à quelques kilomètres. Leur situation financière ne leur permet ni d'accéder au sommet de la tour Eiffel, ni d'acheter une réplique. La tour Eiffel ainsi devient-elle la métaphore de la barrière qui existe entre le centre et les habitants des banlieues.

Les transports en commun jouent un rôle de premier ordre, en tant que cordon reliant la cité au centre. Le RER est la ficelle qui relie la banlieue au monde extérieur. La mère de Taniel dans Les gens du Balto évoque plusieurs fois ses trajets en RER. La plupart de l'action de Mon nerf se déroule dans le RER qui relie Boisy-Fleury et Paris. Mounir va à Paris voir son psychiatre et à la fin du roman il arrive à son but, le terminus Châtelet-Les-Halles.

Lies, le personnage principal de Viscéral, évolue entre deux mondes, voulant sortir de sa cité sans pour autant couper les liens avec ses amis. Lies va à Paris trois fois : pour une session d'audition pour un film, pour le rendez-vous avec le metteur en scène du film et finalement pour le tournage de film. L'antagonisme entre la banlieue et le centre de Paris se voit illustré à travers la perception de Lies, dont le statut du héros lui confère une lucidité critique et lui permet de mesurer le fossé entre les deux rives de Paris. Le monde parisien n'appartient pas aux banlieusards. Dans un ton critique et amer, Lies qualifie le dix-huitième arrondissement de Paris comme « capitale des artistes et des bobards » (Djaïdani 2007 : 102). Il décrit Paris en disant :

Sur la paume du pavé parisien, des muses bronzées déambulent les pas légers, les touristes sont pistes par des pickpockets en tenue de bohème, un paki tente de refourguer sa flore à deux tourtereaux, des bus accordéons brassent l'air, secouant les parasols d'une terrasse branchouillette au compte-gouttes, vu que le prix n’est pas donné. (Djaïdani 2007 : 134)

Le personnage d'Ahlème dans Du rêve pour les oufs est allé le plus loin dans la conquête du territoire parisien. Elle travaille dans un magasin de chaussures qui se trouve à Paris, sur le boulevard de la Chapelle à Barbès. Elle va régulièrement avec ses amis au cinéma et dans des restaurants à Paris. Ahlème se rend à Paris souvent également à cause des rencontres amoureuses avec 
Tonislav. Il lui donne des rendez-vous à la Place d'Italie ou au pied de la fontaine sur la place Carrée à Châtelet-les-Halles. Le personnage principal de Du rêve pour les oufs parvient donc à conquérir et à privatiser l'espace du centre-ville. Michel de Certeau, ethnologue du proche, conçoit la ville comme un texte que les habitants s'approprient et transforment par leur manière de «faire avec » les lieux et d'y passer (1990:231). De Certeau conçoit la marche comme une énonciation puisque l'habitant s'approprie les lieux, les transforme et les actualise en y introduisant sa propre part de subjectivité. Or, Ahlème incarne la figure de l'habitant d'une ville qui transforme l'espace en le pratiquant. Son personnage témoigne de la réduction considérable du fossé qui sépare Paris de ses banlieues.

\section{Les romans de Faïza Guène et Rachid Djaïdani face aux stéréotypes sur la banlieue}

Force est de constater la complexité qui empreint l'image des cités dans les œuvres de Faïza Guène et de Rachid Djaïdani. Ces cités sont, d'un côté, représentées comme des endroits carcéraux et sals et, de l'autre, en tant que villages dans lesquels une vie communautaire et une forte solidarité entre les habitants se sont installées. Dans les romans en question, l'univers des banlieues paraît hétérogène, multiethnique et multiculturel. Les valeurs de métissages et d'interculturalité sont fortement promues. ${ }^{13}$ Les romans de Djaïdani et Guène ont aussi introduit la description des banlieues résidentielles, et ce faisant, ils ont montré qu'il n'est pas judicieux de réduire la notion de banlieue exclusivement aux cités HLM. À travers une multiplicité de regards, ces romans offrent une manière nouvelle de questionner la problématique identitaire de la France contemporaine et de lui donner une perspective inédite en mettant en scène la vie quotidienne des jeunes banlieusards. Les protagonistes des romans de Rachid Djaïdani et de Faïza Guène ne font pas partie du type de jeune délinquant de la banlieue en échec scolaire et au chômage, qui vit pour la violence et le trafic de drogue. Ces romanciers montrent un autre visage des banlieues. Ils racontent aussi la discrimination liée à la géographie d'habitation et les violences dont les jeunes Français, issus de l'immigration et habitant dans les grands ensembles HLM, sont victimes.

Avec leurs représentations des banlieues, ces textes dénoncent leur stigmatisation faite par les médias français. À partir de 1990, la violence est devenue une catégorie dominante d'interprétation des banlieues dans les discours médiatiques. ${ }^{14}$ Le terme de banlieue est devenu une expression commune de ce qui est un problème social. Les reportages journalistiques sur la banlieue insistent sur la misère, le chômage, la délinquance, la crise de l'école, le manque

13 «Quand jétais petite et que maman m’emmenait au bac à sable, aucun enfant ne voulait jouer avec moi. J'appelais ca « le bac à sable des Français ", parce qu'il se trouvait au cœur de la zone pavillonnaire et qu'il y avait surtout des familles des origines françaises qui y habitaient. Une fois, ils faisaient tous une ronde et ils ont refusé de me donner la main parce que c’était le lendemain de l'aïd, la fête du Mouton, et que Maman m'avait mis du henné sur la paume de la main droite. Ces petites têtes à claques croyaient que jétais sale.

Ils n’avaient rien compris à la mixité sociale et au mélange des cultures. En même temps, c'est pas vraiment leur faute. » (Guène 2004 : 89-90)

14 Les soulèvements de Vaulx-en-Velin dans la banlieue lyonnaise en 1990 marquent la reconnaissance officielle de l'existence des banlieues en tant que problème public. 
de perspective d'avenir. Conséquemment, une représentation alarmiste s'est imposée dans les medias et dans le champ politique. Lévocation de l'espace de la banlieue mobilise presque instantanément un réseau de fantasmes et de représentations négatives chez les Français « de souche $»{ }^{15}$

Les romans de Faïza Guène cassent les stéréotypes sur la banlieue et les renversent en introduisant les protagonistes agissant sur leur environnement. Les personnages de Faïza Guène sopposent au statisme et à la victimisation. Ils sont des figures positives dont le comportement et le mode de vie cassent les préjugées et réfutent les stéréotypes sur la banlieue. Les personnages de ses romans sont engagés activement dans la création d'un meilleur avenir personnel. Par exemple, Doria, dans Kiffe kiffe demain, repoussera le déterminisme de son milieu en encourageant les autres protagonistes du roman à se prendre en main à travers les initiatives civiques. Doria n'est plus victime de l'environnement dans lequel elle a grandi. À la fin du roman, elle veut transférer son optimisme au niveau de sa communauté :

Moi, je mènerai la révolte de la cité du Paradis [...] Mais [...] ce sera une révolte intelligente, sans aucune violence, où on se soulèvera pour être reconnus, tous. Il y a pas que le rap et le foot dans la vie (Guène 2004 : 193).

Dans son roman Viscéral, Rachid Djaïdani dessine une voix en contrepoint aux discours sur la banlieue véhiculés par les medias. Djaïdani veut réhabiliter l'image des jeunes habitants des cités, médiatiquement réduits à des motifs stéréotypes. Les romans de Djaïdani et Guène forcent le lecteur à poser un nouveau regard sur les banlieues et ses habitants en dénonçant les stéréotypes et en parlant du racisme dans la société française.

Ses romans remettent en question une relation de hiérarchie entre Paris et sa banlieue en mettant l'accent sur la périphérie et en ignorant la valeur symbolique de Paris. La banlieue est représentée, elle aussi, comme un lieu de production culturel non négligeable. À titre d'exemple, après l'enfermement dans le bunker, Yaz dans Boumkoeur veut écrire un livre. Les romans de Rachid Djaïdani et Faïza Guène introduisent des représentations nouvelles des banlieues dans l'imaginaire collectif. Ils permettent de voir, de l'intérieur, le processus de construction de l'identité urbaine des habitants des banlieues. Leurs romans montrent que les cités sont devenues un espace de création d'identités transnationales, hybrides et multiculturelles.

\section{En guise de conclusion}

Il y a un siècle et demi, une grande diversité des représentations des banlieues parisiennes est mise en circulation par la peinture, la photographie, la bande dessinée, le cinéma et la littérature (Papieau 1996). L'image des banlieues se retrouve, par exemple, dans les romans d'Honoré de Balzac, de Victor Hugo, d'Émile Zola et de Guy de Maupassant. La banlieue est décrite soit comme un endroit malfamé, dangereux et sale, soit comme un endroit de détente, de loisir et

15 Voir Sedel (2009), un livre qui analyse les discours et les pratiques journalistiques et qui retrace l'évolution du traitement journalistique des quartiers populaires. 
de purification du citadin par le retour à la nature. ${ }^{16}$ Toutes ces représentations littéraires montrent l'aspect hybride de la banlieue. La période qui sétend des années soixante-dix aux années quatre-vingts du $\mathrm{XX}^{\mathrm{e}}$ siècle, se traduit par une accélération du rythme de production des représentations. Van Waerbeke (1996: 65), dont le travail démontre la manière dont un capital d'images poétiques des périphéries parisiennes s'est progressivement constitué, souligne qu'une double polarisation s'affirme : d'une part, il est possible de parler de banlieues au quotidien. Il s'agit alors d'un milieu de vécu banal, parfois dur, le plus souvent chaleureusement imprégné de relations familiales, amicales ou amoureuses qui s'y tissent. D’autre part, le pôle opposé est plein d'images noires et négatives. C'est celui des peurs, des angoisses en tous genres ainsi que des situations bloquées.

Marie-Claire Bancquart, dans son étude sur Paris dans la littérature depuis 1945, souligne que le Paris des écrivains parisiens d'après-guerre est décidément une capitale morcelée et changeante (2006 : 351). Les écrivains perçoivent bien la complémentarité et l'unité des deux réalités (la vie citadine à Paris et la vie urbaine en banlieue) qui ne s'opposent pas, mais qui communiquent en permanence et sont assemblées par mille et un fils de nature différente (les transports et les déplacements, le commerce et les distractions, le travail, les amis et la famille, les administrations, etc.).

Les représentations de Paris et de ses banlieues dans les romans de Faïza Guène et Rachid Djaïdani s'inscrivent fortement dans la tradition littéraire hexagonale des représentations de Paris et de ses banlieues en l'élargissant à de nouveaux apports. Leurs romans constituent un nouvel épisode au sein de cette tradition, sans pour autant cesser de témoigner de la transformation des espaces urbain et périurbain parisiens. À travers les visions de Paris et de ses banlieues dans leurs romans, contestant l'image souvent mythifiée et unifiée de la capitale, l'histoire de la France multiculturelle est dessinée. Ces romans enregistrent d'autres images de Paris et de ses banlieues tout en modifiant la notion de banlieue. Les romans de Faïza Guène et Rachid Djaïdani affirment la thèse de Chantal Liaurotzos selon laquelle c'est en marges que se décident et se dessinent à présent les cartographies littéraires de Paris (2007 : 423).

\section{Références bibliographiques}

Bancquart, M.-C. (2006). Paris dans la littérature française après 1945. Paris: Éditions de la Différence. Bronner, L. (2010). La loi du ghetto. Enquête sur les banlieues françaises. Paris: Calmann-Levy. Caillos, R. (1938). Paris, mythe moderne. In Le mythe et l'homme (pp. 150-171). Paris: Gallimard. Charef, M. (1983). Le thé au harem d'Archi Ahmed. Paris: Mercure de France.

Citron, P. (1961). La poésie de Paris dans la littérature française de Rousseau a Baudelaire. Paris: Éditions de Minuit.

De Certeau, M. (1990). L'invention du quotidien. Paris: Gallimard.

Djaïdani, R. (1999). Boumkoeur. Paris: Les Éditions du Seuil.

16 Dans les romans et les nouvelles, les personnages se rendent surtout en banlieue pour des ballades dominicales, afin d'explorer le coté rural de la banlieue, par exemple dans Une partie de campagne et Sur leau de Guy de Maupassant ou dans L'Oeuvre ou Thérèse Raquin d'Émile Zola. 


\section{- (2004). Mon nerf. Paris: Les Éditions du Seuil. \\ - (2007). Viscéral. Paris: Les Éditions du Seuil.}

Frayer, A.; \& Vincent, E. (2011). ZUS: un habitat sur deux est issu de l'immigration. Le Monde, 2 novembre 2011, 11.

Guène, F. (2004). Kiffe kiffe demain. Paris: Hachette Littératures.

- (2006). Du rêve pour les oufs. Paris: Hachette Littératures.

- (2008). Les Gens du Balto. Paris: Hachette Littératures.

Hargreaves, A. G. (1991). Voices from the North African Immigrant Community in France: Immigration and Identity in Beur Fiction. Oxford/New York: Berg.

- (2014). De la littérature 'beur' à la littérature de 'banlieue': des écrivains en quête de reconnaissance. Africultures, 97, 144-149.

Highonnet, P. (1999). Les mythes de Paris, des Lumières au surréalisme. Paris: Collège de France.

Horvath, C. (2007). Le roman urbain contemporain en France. Paris: Presses Sorbonne Nouvelle.

Laronde, M. (1993). Autour du roman beur. Paris: Harmattan.

Papieau, I. (1996). La construction des images dans les discours sur la banlieue parisienne : pratiques et production esthétiques. Paris: Harmattan.

Pinçonnat, C.; \& Liaroutzos, C. (2007). Paris, cartographies littéraires. Paris: Le Manuscrit.

Ronai, S. (2004). Paris et sa banlieue: je t'aime, moi non plus. Hérodote, revue de géographie et géopolitique, $113,28-47$.

Sedel, J. (2009). Les medias \& La banlieue. Paris: Le Bord de l'eau.

Stierle, K. (2002). La capitale des signes. Paris et son discours. Paris: Éditions de la Maison des sciences de l'homme.

Tarr, C. (2005). Reframing difference. Beur and banlieue filmmaking in France. Manchester: Manchester University Press.

Van Waerbeke, J. (1996). La poétique spatiale des représentations de la banlieue de Paris. Géographie et Cultures, 19, 51-78.

Westphal, B. (2000). Pour une approche géocritique des textes. Esquisse. In La géocritique mode demploi (pp. 9-39). Limoges: Presses universitaires de Limoges.

—. (2007). La géocritique. Réel, fiction, espace. Paris: Les Éditions de Minuit. 
